\title{
Concepções e autorregulação da aprendizagem da arte
}

\author{
Luiz Gustavo Lima Freire \\ Faculdade dos Guararapes Laureate International Universities
}

\section{Resumo}

Este artigo tem três objetivos. O primeiro, que procuramos implementar na primeira seção, consiste numa breve revisão dos estudos sobre concepções de e autorregulação da aprendizagem em geral. O segundo, que tentamos alcançar nas duas seções seguintes, é o de abordar a investigação daquelas duas variáveis no caso particular da aprendizagem das artes. Finalmente o terceiro objetivo, relacionado à quarta seção, consiste numa sucinta revisão dos estudos que têm procurado relacionar essas duas linhas de investigação.

Palavras-chave: Arte-educação; autorregulação; aprendizagem.

\section{Conceptions and self-regulations on art learning}

\begin{abstract}
This paper has three goals: the first goal seeks to implement in the first section, a brief review of studies on conceptions of learning and selfregulation in general. The second goal is to investigate these two variables in the particular case of learning arts. The third objective, related to the fourth section, is a brief review of studies that have sought to relate these two lines of investigation.
\end{abstract}

Keywords: Art education; self regulation; learning.

\section{Concepciones y autorregulación del aprendizaje del arte}

\section{Resumen}

Este artículo tiene tres objetivos. El primero, que buscamos implementar en la primera sección, consiste en una breve revisión de los estudios sobre concepciones de y autorregulación del aprendizaje en general. El segundo, que tentamos alcanzar en las dos secciones siguientes, es el de abordar la investigación de aquellas dos variables en el caso particular del aprendizaje de las artes. Finalmente el tercer objetivo, relacionado a la cuarta sección, consiste en una breve revisión de los estudios que ha buscado relacionar esas dos líneas de investigación.

Palabras-clave: arte-educacion; autorregulación; aprendizaje. 


\section{Introdução}

A Psicologia Educacional, interessada na aprendizagem dos estudantes, tem se desenvolvido em torno de diferentes perspectivas teóricas. Uma dessas perspectivas diz respeito à forma como os estudantes autorregulam a sua aprendizagem ("Self-Regulated Learning" - SRL). Outra perspectiva se centra no modo como eles abordam a aprendizagem, combinando concepções de aprendizagem, motivação e estratégias de aprendizagem ("Students' Approaches to Learning" - SAL). Particularmente quando trata das concepções de aprendizagem, a teoria SRL utiliza uma perspectiva dita "fenomenográfica". Ora, embora estas perspectivas - distintas mas relacionáveis - tenham se desenvolvido sobretudo no domínio da aprendizagem em geral, alguns estudos se debruçam sobre as variáveis que interessam a ambas em domínios específicos de aprendizagem - como é o das artes. Alguns destes estudos partem da noção de que provavelmente existe uma variação na forma como os estudantes concebem a aprendizagem da arte e de que estes podem autorregular essa mesma aprendizagem. Hipoteticamente, o tipo de concepção de aprendizagem da arte está relacionado à frequência e ao tipo de autorregulação dessa mesma aprendizagem (Douglas, 1996; Freire, \& Duarte, 2012; Martínez-Fernández, 2007; Purdie, Hattie, \& Douglas, 1996).

\section{Concepções e autorregulação da aprendizagem}

Os estudos fenomenográficos se centram no modo como os estudantes representam a aprendizagem. Realça-se que os estudantes se comportam não apenas em função dos dados objetivos do processo de ensino-aprendizagem, mas em função das suas concepções de aprendizagem, definidas como o significado que os indivíduos atribuem a tal fenómeno. Através da análise dos discursos e do estabelecimento de taxonomias, procura-se mapear os modos de representar a aprendizagem com vistas a proporcionar intervenções que possibilitem mudanças nas formas de as pessoas aprenderem (Freire, \& Duarte, 2010; Marton, \& Säljö, 1976).

Segundo a perspectiva fenomenográfica, existem basicamente duas formas de os indivíduos representarem (conceberem) a aprendizagem: a quantitativa e a qualitativa. A primeira se caracteriza pela noção de aprendizagem enquanto memorização, recolha e acumulação mecânica das informações, e a segunda, enquanto compreensão, construção e abstração das informações, com consequente desenvolvimento pessoal (Biggs, 2006). Paralelamente foram descritas concepções de aprendizagem mais específicas, que são variantes das duas anteriores e definem aquele fenómeno como aumento de conhecimento; memorização e reprodução; memorização e aplicação; compreensão; reinterpretação; ou mudança pessoal (Marton, Dall'Alba, \& Beaty, 1993).
As concepções de aprendizagem qualitativas estão normalmente associadas a uma abordagem profunda da aprendizagem (i.e., combinação de uma motivação intrínseca com uma estratégia de compreensão) e a melhores resultados acadêmicos. Tendem a produzir uma melhor compreensão dos conteúdos e não excluem necessariamente a memorização. Elas podem resultar de contextos que, entre outras coisas, desenvolvam o espírito crítico, através da exposição a diferentes perspectivas do conhecimento (Marton, \& Booth, 1997; Yang, \& Tsai, 2010).

As concepções de aprendizagem não são "retratos" da experiência pessoal, porque não se referem aos modos particulares de aprender, mas ao que se pensa sobre a aprendizagem em geral. Elas possuem uma natureza relacional, e não são apenas características do estudante ou do contexto, mas se estabelecem na relação entre os dois. Dizem respeito ao modo característico de o estudante abordar a aprendizagem (estudar), aos seus aspectos pessoais (cognitivos, afetivos, interpessoais), aos aspectos do contexto (conteúdos, métodos, materiais, objetivos, recursos educativos, ambientes físicos e socioculturais, etc.) e aos resultados escolares (Hernández Pina, \& Sánchez, 2011; Marton, 1981).

As concepções de aprendizagem se referem aos aspectos gerais do aprender, por isso ultrapassam a natureza das tarefas acadêmicas específicas, sendo possível refletirmos sobre a forma como elas se exprimem relativamente a algumas atividades particulares. Na Faculdade de Psicologia da Universidade de Lisboa tem-se empreendido nos últimos anos uma linha de investigação com o objetivo de compreender as concepções de aprendizagem em geral e para diferentes atividades acadêmicas. Nesse âmbito, foram desenvolvidos estudos que pretenderam compreender as concepções de aprendizagem em geral (Duarte, 2004) de estudantes universitários brasileiros (Freire, \& Duarte, 2010) para as situações de avaliação (Rendeiro, \& Duarte, 2007) e para a aprendizagem com o computador (Rebelo, \& Duarte, 2012).

Paralelamente à investigação conduzida pela Fenomenografia, estudos realizados noutro enquadramento teórico têm demonstrado que, para aprender, os estudantes podem ser mais heterorregulados (orientados por padrões externos) ou autorregulados (orientados por padrões pessoais). A autorregulação da aprendizagem pode ser definida como o exercício de influência que o indivíduo impõe à própria motivação, aos processos de pensamento, aos estados emocionais e aos padrões de comportamentos implicados na aprendizagem. Ao exercer esse controle ele adota padrões internos, monitora as suas ações e utiliza incentivos para mobilizar e sustentar os seus esforços e para cumprir os seus próprios objetivos (Escudero, Carreto, \& Garcia, 2010; Palacio, Álvarez, \& Dorantes, 2010; Zimmerman, 1998, 2000, 2008).

A aprendizagem autorregulada pode ser desenvolvida num contexto favorável. Ela não deve ser encarada como uma disposição, mas como um processo de autodireção, através do qual as aptidões mentais são transformadas em 
competências acadêmicas. No Ensino Superior, que é um contexto especialmente não estruturado (comparativamente aos anos de estudo anteriores), pode-se considerar que o sucesso acadêmico é mais dependente desse processo, ou seja, da habilidade do estudante para ser mais autônomo (Cerezo, Núñez, Fernández, Fernández, \& Tuero, 2011; García-Ros, \& Pérez-González, 2011; Hernández Pina, Fonseca, Rosário, \& Tejada, 2010; Miñano, \& Castejón, 2011; Rosário e cols., 2010).

Diversos autores têm demonstrado que a aprendizagem autorregulada é um processo cíclico que se desenvolve em três fases interdependentes e eventualmente concomitantes (Horta, 2010; Rosário e cols., 2010; Zimmerman, 1998): antecipação - criação de objetivos e seleção de estratégias para alcançá-los; controle volitivo - monitoramento da volição (automonitoramento) de modo a atingir as metas propostas; e autorreflexão - avaliação da produção acadêmica feita através da comparação da informação que foi monitorada com o objetivo concreto de uma atividade.

A aprendizagem autorregulada tem recebido a contribuição de inúmeras investigações. Ela tem sido relacionada, por exemplo, às abordagens à aprendizagem (Van den Brink, 2006), mas uma revisão da literatura específica permitiu constatar a escassez de pesquisas que busquem compreender a forma como ela se relaciona às concepções de aprendizagem. Os poucos estudos que existem (Douglas, 1996; Martínez-Fernández, 2007; Purdie, Hattie, \& Douglas, 1996) sugerem uma correlação positiva entre as concepções qualitativas e uma aprendizagem mais autorregulada, e uma ausência de correlação entre esse tipo de aprendizagem e as concepções quantitativas.

Por outro lado, embora a perspectiva fenomenográfica e a da aprendizagem autorregulada tenham se interessado, sobretudo e correspondentemente, pela concepção que os estudantes têm da aprendizagem e pela forma como a autorregulam em geral, alguns estudos se orientam para a pesquisa destas representações e destes processos no caso específico da aprendizagem na área das artes. As duas seções que se seguem reveem precisamente alguns dos estudos realizados com esse intuito.

\section{As concepções de aprendizagem das artes}

Enquanto abundantes para as concepções de aprendizagem em geral, são escassos os estudos interessados nestas representações no caso particular da aprendizagem da arte. Nesse contexto, Basto (2013) desenvolveu uma investigação com o objetivo de mapear as concepções de aprendizagem da dança clássica e contemporânea de um grupo português de estudantes do $11^{\circ}$ e $12^{\circ}$ anos de escolaridade. Através de entrevistas semiestruturadas a autora identificou a existência de concepções de aprendizagem da dança comparáveis às concepções de aprendizagem em geral desveladas anteriormente pela investigação fenomenográfica. Com efeito, a aprendizagem da dança poderia ser concebida como um processo de aquisição de conheci- mentos, de técnicas de dança, de conhecimentos de dança progressivamente complexos, ou como memorização de movimentos; como desenvolvimento artístico ou pessoal ou como compreensão da evolução do domínio artístico ou do próprio funcionamento corporal, e como uma conjugação desses processos. Foram identificadas ainda concepções aparentemente novas, que representavam a aprendizagem da dança como uma expressão através do movimento, e um processo baseado na perceção, na automotivação, na visualização, na execução ou no ensino da dança a colegas.

Torrado e Pozo (2011) desenvolveram um importante estudo com o objetivo de analisar a forma como professores de música de conservatórios profissionais concebiam a aprendizagem dos seus alunos. Os autores desenvolveram um questionário de teorias implícitas sobre a aprendizagem de instrumentos musicais composto por vinte perguntas, cada uma delas sobre uma etapa ou situação (conhecimento prévio, motivação, organização do ensino, estratégias didáticas, melhora do ensino) que exigia que se tomasse ou valorasse uma decisão problemática de acordo com três alternativas de respostas, as quais representariam as suas teorias implícitas (direta, interpretativa e construtiva). Esse estudo permitiu constatar a existência de três teorias implícitas sobre a aprendizagem da música, designadas de teoria direta, teoria interpretativa e teoria construtiva. Na teoria direta os resultados da aprendizagem são peças disjuntas que se acumulam sumativamente, ou seja, uma nova aprendizagem não afetaria as anteriores. $\mathrm{Na}$ teoria interpretativa os resultados se apresentam como um processo que ocorre através do tempo, porém os componentes básicos (condições; ações observáveis e processos mentais do aprendente; resultados, metas ou conteúdos) da aprendizagem são articulados como elos de uma cadeia causal, linear e unidirecional. Na teoria construtiva a aprendizagem é um sistema dinâmico autorregulado que articula condições, processos e resultados (Pozo, Scheuer, Mateos, \& Echeverría, 2011).

Os resultados obtidos demonstraram que metade das respostas conferidas (50\%) correspondia às posições construtivas, uma grande parte (39\%) às posições interpretativas e uma pequena $(11 \%)$ às posições diretas. As construtivas foram menos frequentes em algumas dimensões ou âmbitos do que em outros. Juntas, as interpretativas e as construtivas superaram oitenta por cento $(80 \%)$ das respostas.

As posições interpretativas foram mais acentudamente predominantes nas estratégias didáticas e as construtivas predominaram na motivação, na organização e na melhora do ensino, tendo a mesma predominância nos conhecimentos prévios. Em todas as etapas ou situações as respostas que aludiam a uma posição direta não alcançaram mais de quinze por cento (15\%). Além disso, foi possível constatar que todos os professores apresentaram respostas correspondentes a mais de uma concepção de aprendizagem.

As concepções de aprendizagem encontradas nesse estudo podem ser consideradas similares às descobertas pela Fenomenografia. Com efeito, a teoria direta parece ser equivalente a uma concepção quantitativa (aumento de conhecimento), e as teorias interpretativa e construtiva, a uma 
concepção qualitativa. A primeira é equivalente à versão reinterpretação e a segunda, à versão mudança pessoal.

Scheuer, Pozo, de la Cruz e Echenique (2011) desenvolveram um estudo com o objetivo de descrever os modos de pensar a aprendizagem do desenho figurativo e explicar o seu desenvolvimento. Dada a carência de investigações nessa área, essa pesquisa se reveste de grande interesse, motivo pelo qual passaremos a descrevê-la em pormenores. Para estudar as concepções das crianças sobre a aprendizagem do desenho de acordo com as teorias implícitas, os autores empregaram como esquema organizador uma formulação que considerou a aprendizagem a partir de três componentes: as condições, as ações observáveis e os processos mentais do aprendente e os resultados, metas ou conteúdos da aprendizagem.

Baseados nesse esquema eles construíram um guião estruturado de entrevista individual. Posteriormente eles propuseram diversas tarefas verbais e gráficas relativas à intervenção desses três componentes, tanto no que concerne à aprendizagem dos próprios estudantes quanto ao que estes pensavam sobre a dos demais.

Nesse estudo foram entrevistadas crianças argentinas de quatro, cinco e seis anos de idade. As respostas às entrevistas foram submetidas a uma análise de conteúdo, que permitiu discriminar duas concepções da aprendizagem do desenho, correspondentemente designadas de teoria direta (com duas versões) e de teoria interpretativa, que a seguir se descrevem.

A teoria direta, focalizada nos resultados acumulativos da aprendizagem e expressa por alguns estudantes de quatro anos de idade, caracterizava-se por uma concepção de aprendizagem do desenho enquanto ampliação de resultados gráficos. Esta concepção é acumulativa e dicotómica, e não integra matizes epistêmicas. Ela se centra nos produtos da aprendizagem concebidos como realizações inconexas e sumativas (saber mais desenhos), de modo que para efetuar tais realizações seriam necessárias apenas atividades básicas e manifestas (desenhar e copiar modelos).

Foi identificada uma versão da teoria direta - a agência do entorno. Essa concepção foi apresentada pelos estudantes dos três grupos de idade. Ela articula condições e resultados de aprendizagem de acordo com uma lógica linear, ou seja, se as condições se cumprem, a aprendizagem se produz inevitavelmente, ao passo que se a aprendizagem for constatada, o motivo será que as condições foram cumpridas. Os fatores (condições) são o crescimento e a saúde do aprendente, sua motivação para aprender e um ambiente que ofereça o ensino e os modelos de produtos e de procedimentos de desenho, ou seja, a conjugação das atividades básicas do aprendente (desenhar e copiar), com o cumprimento das condições necessárias a sua execução, seria suficiente para assegurar os resultados - concebidos, como na versão anterior, como acumulação sumativa de novidades. Aprender a desenhar é adquirir os modos que as pessoas mais experientes nessa área utilizam - pessoas que estruturam uma relação assimétrica de ensino, guiam a atividade de quem aprende e emergem como sujeitos da aprendizagem (do ponto de vista postural e motor). A aprendizagem é uma consequência de fatores socioculturais (ensino deliberado, acesso a modelos) e biológicos (crescimento e saúde).

Por seu lado, a teoria interpretativa foi expressa por crianças de cinco e seis anos de idade. Esse grupo articulou os três componentes da aprendizagem com foco nas representações e nos processos mentais de quem aprende. $O$ aprendente se constitui no agente do processo de aprendizagem, não apenas através do exercício da sua atividade observável (desenhar, ver e copiar modelos), mas também pela geração e ativamento das suas representações internas (registrar, recordar, antecipar, compreender) e pelo exercício da autorregulação sobre as condições e o curso da sua ação (planejar metas, ajustar-se a sua execução, avaliar os próprios resultados e utilizar deliberadamente instrumentos de apoio, como réguas ou modelos).

A aprendizagem faz avançar o modo de desenhar, assim como gera novas representações mentais. Apesar disso, essa teoria não pode ser considerada uma versão da construtiva, uma vez que não existe referência à complexidade. A atividade mental alcança somente a geração de representações mentais novas relativamente às situações e aos objetos externos ou à ativação das representações existentes.

No geral, nos resultados do estudo de Scheuer, Pozo, de la Cruz e Echenique (2011) foi constatado que as concepções de aprendizagem do desenho evoluem de uma teoria direta a uma interpretativa da agência e que tal evolução pode ser favorecida pela capacidade reflexiva; também, aqui podemos constatar uma semelhança com as concepções propostas pelo referencial fenomenográfico. A teoria direta seria semelhante à concepção quantitativa, enquanto a interpretativa à qualitativa.

\section{Aprendizagem autorregulada das artes}

A perspectiva da aprendizagem autorregulada, geralmente orientada primariamente para esta aprendizagem, também não deixa de se interessar pela relação da autorregulação com a aprendizagem da arte.

De acordo com Catterrall (2005), a influência da aprendizagem da arte se manifesta a nível cognitivo quando as habilidades (artísticas) provocam impactos nas estruturas cognitivas já existentes ou conduzem ao desenvolvimento de novas estruturas e a nível afetivo, quando esta influência promove o compromisso pessoal do estudante (o seu interesse e persistência nas tarefas) e a atribuição do êxito ao desempenho eficaz.

O estudante que experiencia uma aprendizagem artística integradora prova continuamente suas capacidades, questiona-se, avalia e modifica sua forma de enfrentar as distintas tarefas que enfrenta, toma consciência das suas debilidades e recorre a novos (mais) esforços em momentos críticos da sua aprendizagem (Soto, \& Macías, 2011). 
Segundo Padilla (2007), o estudante de desenho precisa saber que avança fragmentariamente, mediante tentativas, erros e ajustes subordinados à comprovação e à avaliação dos objetivos iniciais, mediante a execução de procedimentos para alcançar os objetivos (resultados) e eventuais reordenamentos, caso os objetivos iniciais não tenham sido atendidos.

Ora, se assumirmos esse pensamento, os comportamentos futuros não têm como base o acaso, mas um processo cujas etapas são análogas às que podemos encontrar no decurso da aprendizagem autorregulada proposta por Zimermam (1998, 2000): planejamento (antecipação), execução (controle volitivo ou automonitorização) e autorreflexão.

Procurando integrar os fundamentos da autorregulação na aprendizagem do desenho, estabelecemos um emparelhamento das características da aprendizagem da arte propostas por Eisner (2004) com as fases da autorregulação oferecidas por Zimmerman (2000). A seguir pode-se consultar um quadro com uma síntese dos resultados desse trabalho.
Assim como Zimmerman (2000), Eisner (2002) ressaltou a importância dos objetivos e da motivação quando afirmou:

[É Preciso] ajudar os estudantes a criarem um profundo sentido de finalidade que sirva como motivador principal do seu trabalho. (...) um objetivo que thes importe genuinamente. (...) grande parte do que os estudantes fazem na escola, fazem para adaptar-se às tarefas dadas pelo professor (...) para adaptar-se às expectativas externas. Mas o trabalho nas artes requer justamente o contrário. Requer que se tenha algo a dizer, algo dentro que necessite ser expressado (p. 48).

Segundo ele, no ato de criação artística são ainda importantes os objetivos não previstos inicialmente:

No transcurso do trabalho podem aflorar e descobrir-se objetivos, estes não têm que estar planificados de antemão. São fontes de surpresa e constituem oportunidades

Quadro 1. Análise conceitual da relação entre algumas das características da aprendizagem da arte (Eisner, 2004) e as fases da autorregulação (Zimmerman, 2000).
Fases da auto-regulação
Aprendizagem da arte

Antecipação

(Estabelecimento de objetivos e selecão das estratégias para alcançálos).

- Planificação do trabalho relativo à obra de arte (formulação de objetivos relacionados ao estabelecimento ou materialização de uma ideia).

- Processo de seleção dos materiais necessários à execução da obra de arte (baseados na avaliação da sua adequação e qualidade).

- Análise das cores e das texturas a serem materializadas.

- Execução das obras.

Controle volitivo

(Execução e monitoramento dos procedimentos que estão a ser usados para atender os objetivos).
- Criação do veículo imaginativo.

- Aplicação do repertório técnico (sistema de procedimentos dinâmico, uma vez que, se constitui numa oportunidade para a revisão, o descobrimento e a alteração dos próprios instrumentos).

- Processo de resolução ou de execução da obra propriamente dito.

\section{Auto-reflexão}

(Avaliação da produção acadêmica baseada no atendimento dos objetivos, mas não só).
- Comparação dos trabalhos (da obra de arte) com a intenção de verificar o grau de atendimento dos objetivos que haviam sido propostos. Essa comparação pode ser feita com base nos modelos (nas obras de arte e na opinião das outras pessoas sobre essas mesmas obras). 
inesperadas, que frequentemente podem introduzir mudanças no curso do trabalho. Sem embargo, a expressão implica a possessão de um objetivo prévio à ação sobre um material (p. 49).

Matos, Pineda e Vásquez (2010), ao proporem um modelo de aprendizagem e de ensino das artes baseado nos fundamentos de Vigotsky, também realçaram a importância da intencionalidade (ou da autorregulação).
Com efeito, nos processos de criação também aprendemos a tomar decisões. Toda criação envolve mais uma atividade de exploração, invenção e tomada de decisão do que de conformismo às regras. $O$ fato de o estudante avaliar a adequação e a qualidade dos materiais e suportes e de analisar as cores e as texturas a serem materializadas exige um grande esforço do pensamento visual (Meira, \& Pillotto, 2011).

Quadro 2. Estratégias de aprendizagem autorregulada do curso de Soto e Macías (2011).

\begin{tabular}{|c|c|c|}
\hline & Área & Estratégia a ser utilizada pelo aluno \\
\hline \multirow{7}{*}{$\begin{array}{l}\text { Previsão, } \\
\text { planificação e } \\
\text { ativação }\end{array}$} & \multirow{4}{*}{ Cognitiva } & $\begin{array}{l}\text { Análise do conhecimento novo e a sua relação com os } \\
\text { anteriores. }\end{array}$ \\
\hline & & $\begin{array}{l}\text { Estabelecimento de metas de acordo com a especificidade, a } \\
\text { proximidade e a dificuldade. }\end{array}$ \\
\hline & & $\begin{array}{l}\text { Definição de estratégias de acordo com as metas. } \\
\text { Identificação de eventuais problemas e como resolvê-los. }\end{array}$ \\
\hline & & $\begin{array}{l}\text { Decisão sobre a utilização de estratégias (tomada de } \\
\text { apontamentos, discussão das dúvidas com os colegas, pedido } \\
\text { de apoio específico, etc.). }\end{array}$ \\
\hline & \multirow[b]{2}{*}{ Motivacional } & $\begin{array}{l}\text { Determinação do prazer que se tem com o conteúdo ou tarefa, } \\
\text { e o valor atribuído. }\end{array}$ \\
\hline & & $\begin{array}{l}\text { Identificação das crenças acerca das próprias capacidades } \\
\text { para aprender ou executar a tarefa, o que é fácil ou difícil, o que } \\
\text { se compreende e o que não, de acordo com o conhecimento } \\
\text { que se tem e de acordo com as habilidades e experiencias } \\
\text { (percepção de auto-eficácia). }\end{array}$ \\
\hline & Contextual & $\begin{array}{l}\text { Percepção sobre as aulas, características e comportamentos } \\
\text { do professor e dos colegas. }\end{array}$ \\
\hline \multirow{3}{*}{ Supervisão } & Cognitiva & $\begin{array}{l}\text { Identificação dos conhecimentos que se sabe, dos que se } \\
\text { acabou de saber e dos que se compreendeu. }\end{array}$ \\
\hline & \multirow[b]{2}{*}{ Motivacional } & $\begin{array}{l}\text { Identificação das motivações básicas orientadas às metas } \\
\text { (definidas nas sessões anteriores) e aos motivos das tarefas. }\end{array}$ \\
\hline & & $\begin{array}{l}\text { Identificação das crenças sobre as próprias capacidades para } \\
\text { aprender ou para executar a tarefa, com base no conhecimento } \\
\text { novo, na execução da tarefa e nos resultados conquistados } \\
\text { (observados nas sessões anteriores). }\end{array}$ \\
\hline \multirow{3}{*}{ Controle } & \multirow{3}{*}{$\begin{array}{l}\text { Cognitiva, motivacional e } \\
\text { comportamental }\end{array}$} & $\begin{array}{l}\text { Adaptação ou realização de mudanças das atividades segundo } \\
\text { as próprias cognições (atividades cognitivas e meta-cognitivas). }\end{array}$ \\
\hline & & $\begin{array}{l}\text { Realização de ajustes segundo as avaliações dos resultados } \\
\text { (administração do tempo e redirecionamento do esforço). }\end{array}$ \\
\hline & & Adaptação ou mudança na auto-eficácia e na auto-motivação. \\
\hline Reação e reflexão & $\begin{array}{l}\text { Cognitiva, motivacional, } \\
\text { comportamental e contextual }\end{array}$ & $\begin{array}{l}\text { Desenvolvimento de juízos, atribuições e auto-avaliações do } \\
\text { próprio funcionamento, de modo a poder regular a motivação, o } \\
\text { comportamento e o contexto. }\end{array}$ \\
\hline
\end{tabular}


Winner e Hetland (2006) demonstraram, através de um estudo cujo objetivo foi determinar os efeitos do ensino-aprendizagem das artes visuais numa escola de bacharelado, que os estudantes podem aprender: 1- a desenvolver a sua técnica (como e quando utilizar instrumentos e materiais); 2- a persistir nas tarefas (atender aos projetos durante um longo período); 3- a imaginar (gerar imagens mentais que os ajudem a antecipar e orientar as suas obras); 4- a expressar-se (conquistar uma visão e significado pessoal em seu trabalho); 5- a observar (notar as qualidades dos objetos, tais como detalhes, brilhos, texturas, cores, estilos, etc.); 6- a refletir (sobre as suas obras e as dos demais, questionando e explicando as suas intenções e decisões durante o seu processo de elaboração); 7- a experimentar (ir além do que se havia feito correndo riscos); 8- a compreender o conteúdo (a história da Arte e como ela se relaciona com o mundo).

Em nossa opinião, os resultados desse estudo poderiam querer demonstrar também que a aprendizagem da arte pode favorecer a autorregulação da aprendizagem, pois muito do que foi aprendido pelos alunos pode ser confundido com os aspectos autorregulatórios, como é o caso da persistência nas tarefas e a antecipação e a orientação de objetivos.

Soto e Macías (2011) desenvolveram uma investigação sobre os efeitos do ensino-aprendizagem da arte. O estudo foi realizado com estudantes universitários de Ciências Sociais e Humanidades e teve como objetivo determinar se um curso para a aprendizagem das artes visuais desenhado com base num modelo de autorregulação poderia propiciar mudanças significativas na motivação e nas estratégias de aprendizagem autorregulada.

O curso foi definido a partir de elementos pedagógicos e conteúdos temáticos de desenho, de escultura e de experiências instrucionais segundo a abordagem teórica de Pintrich (2000). Esperava-se que depois dele os estudantes tivessem aprendido a autorregular a sua aprendizagem. A seguir, apresenta-se um quadro onde se pode verificar quais eram as estratégias de autorregulação que os estudantes deveriam aprender durante o curso, de modo que pudessem utilizá-las também nas demais disciplinas escolares.

Especificamente, foi demonstrado que as áreas mais favorecidas pelo curso foram as relacionadas ao desenvolvimento da obra (área cognitiva - autorregulação metacognitiva e busca de ajuda) e à motivação (autoeficácia e valor intrínseco da tarefa). Com efeito, foi evidenciado que a educação artística integrada tem o potencial de desenvolver a cognição (autonomia e reflexão) dos estudantes, constituindo-se num meio de expressão do pensamento complexo e criativo e das sensações e emoções do aprendente, sobretudo se for enriquecida com os fundamentos da aprendizagem autorregulada.

Como constatado na análise das duas seções anteriores, tanto a investigação fenomenográfica sobre as concepções de aprendizagem dos estudantes como a investigação sobre a aprendizagem autorregulada interessam-se, embora ainda periférica e separadamente, pela aprendizagem no domínio específico das artes. Urge assim, por um lado, expandir o conhecimento de como aquelas variáveis se comportam neste domínio, e por outro, explorar como essas mesmas variáveis se relacionam. É precisamente com estes dois objetivos que se desenvolveu o projeto de investigação que a seguir se apresenta.

\section{Concepções de autorregulação da aprendizagem}

Uma revisão da literatura específica permitiu constatar que, apesar de teoricamente consistentes, essas duas linhas de investigação ( $S A L$ - Student Approaches to Learning e SRL e Self-regulated Learning) carecem de estudos empíricos que integrem suas contribuições. No âmbito da aprendizagem das artes não parecem existir pesquisas com esse objetivo ${ }^{1}$. Esse empreendimento possivelmente ajudaria a pensar a educação artística de maneira diferente, provocando um deslocamento das preocupações relacionadas ao modo como se ensina para o modo se aprende a arte.

Os poucos estudos desenvolvidos com o objetivo de relacionar as concepções à autorregulação da aprendizagem em geral sugerem a fundamentalidade das concepções, uma vez que se assume que elas influenciam, direta e/ou indiretamente, a autoeficácia, as atribuições para o sucesso académico e a utilização de estratégias cognitivas (Ferla, Valcke, \& Schuyten, 2008).

Rosário e Almeida (1999) realizaram uma investigação em estudantes do $10^{\circ}$ e $12^{\circ}$ ano do Ensino Secundário Português com o objetivo de compreender as suas concepções de aprendizagem e a relação com as estratégias de autorregulação dessa mesma aprendizagem. Nesse sentido, uma amostra de 558 alunos foi avaliada de acordo com uma metodologia qualitativa assente nos estudos de Zimmerman, B. e Martinez-Ponz, M. (1986), os quais identificaram catorze estratégias de autorregulação de aprendizagem, e de Marton e colaboradores, que desvelaram concepções de aprendizagem quantitativas e qualitativas.

Os resultados obtidos revelaram que os estudantes com concepções de aprendizagem qualitativas, se comparados àqueles com concepções quantitativas, além de melhores classificações escolares, apresentavam uma média mais elevada relativamente às estratégias de procura de informação, e de procura de ajuda de adultos, enquanto no tocante às outras estratégias não pareciam apresentar diferenças estatisticamente significativas.

Assim, pode-se assumir que as exigências percebidas do contexto escolar podem despoletar comportamentos de estudo coerentes não apenas com as concepções de

1 Enquadrado no Programa de Investigação em Concepções de e Abordagens à Aprendizagem da Faculdade de Psicologia da Universidade de Lisboa (https://sites.google.com/site/ programainvestigacao) estamos desenvolvendo um estudo, no âmbito de um doutoramento, com o objetivo de mapear as concepções de aprendizagem do desenho artístico, caracterizar as estratégias de autorregulação dessa mesma aprendizagem e estudar a relação entre os tipos de concepções e a frequência/tipos de estratégias de autorregulação. 
aprendizagem, mas também com os objetivos escolares desenhados pelos estudantes.

Martínez-Fernández (2007), num estudo que pretendeu relacionar as concepções de aprendizagem às estratégias metacognitivas em estudantes de Psicologia, verificou a existência de uma correlação positiva entre concepções de aprendizagem "qualitativas" e estratégias metacognitivas, enquanto, pelo contrário, não observou relação alguma entre concepções "quantitativas" e estratégias metacognitivas.

Purdie e colaboradores (1996), por sua vez, num estudo que objetivou analisar a relação entre concepções e estratégias de aprendizagem, constataram que, no geral, os estudantes que possuem uma concepção de aprendizagem "qualitativa" tendem a utilizar mais estratégias de aprendizagem.

\section{Conclusão}

Embora ainda escassos, os estudos sobre a forma como os estudantes representam a aprendizagem da arte e sobre o modo como regulam essa mesma aprendizagem permitem começarmos a ter um esboço de como estas duas variáveis se comportam. Desde já, os resultados destes estudos sugerem, por um lado, que os estudantes podem representar de formas diversificadas a aprendizagem no domínio das artes: por um lado, podem entendê-la como um processo mais cumulativo ou mais compreensivo, por outro, podem confrontar a aprendizagem das artes de forma mais ou menos autorregulada, mobilizando uma variedade de estratégias de aprendizagem.

Relativamente à forma como essas duas linhas de investigação se comportam (relacionam) no caso específico da aprendizagem das artes, seria interessante averiguar se, a exemplo do que ocorre na aprendizagem em geral, as concepções "qualitativas" (i.e., aprendizagem enquanto compreensão e/ou implicando uma mudança pessoal) estariam mais associadas a uma regulação interna da aprendizagem (autonomia para aprender) e a mais e melhores estratégias de autorregulação da aprendizagem.

Seria igualmente interessante averiguar se as concepções "quantitativas" (i.e., aprendizagem enquanto memorização e/ou reprodução do conhecimento) estariam mais associadas a uma regulação externa (menor autonomia para aprender) e a uma utilização reduzida de estratégias de aprendizagem autorregulada, sendo estas, nesse caso, menos elaboradas.

Parece-nos necessário testar a possível confirmação desta imagem inicial com mais estudos sobre a aprendizagem das artes que igualmente procurem relacionar concepções de aprendizagem e autorregulação e assim contribuam para compreender e melhorar a forma como os estudantes aprendem nesta área disciplinar.

Os resultados desses estudos serão relevantes porque essa relação pode influenciar o processo e os resultados acadêmicos. Tais resultados podem contribuir para a qualidade desses mesmos processos e produtos, através do desenvolvimento de eventuais intervenções sobre as concepções e as estratégias de autorregulação da aprendizagem do desenho artístico.

\section{Referências}

Basto, M. (2013). Conceções de aprendizagem da dança em estudantes de dança clásica e contemporânea. Dissertação de Mestrado, Faculdade de Psicologia da Universidade de Lisboa, Lisboa.

Biggs, J. (2006). Calidad del aprendizaje universitario. Madrid: Narcea.

Catterall, J. (2005). Conversation and silence: transfer of learning through the arts. Journal for Learning through the Arts: A Research Journal on Arts Integration in Schools and Communities, 1 (1), $1-12$

Cerezo, R., Núñez, J., Fernández, E., Fernández, N., \& Tuero, E. (2011). Programas de intervención para la mejora de las competencias de aprendizaje autorregulado en educación superior. Revista Perspectiva Educacional, 50 (1), 1-30.

Douglas, G. (1996). Student conceptions of learning and their use of self-regulated learning strategies: a cross-cultural comparison. Journal of Educational Psychology, 88 (1), 87-100.

Duarte, A. (2004). Concepções de aprendizagem em de estudantes universitários Portugueses(as). Psicologia, 18(1), 147-163.

Eisner, E (2002). Ocho importantes condiciones para la enseñanza y el aprendizaje en las artes visuales. Arte, Individuo y Sociedad, № Extra 1, 46-56.

Eisner, E (2004). El arte y la creación de la mente : el papel de las artes visuales en la transformación de la conciencia (G. Sánchez, Trad.). Barcelona: Paidós Ibérica.

Escudero, P., Carreto, M., \& Garcia, A. (2010). Motivación y autorregulación a partir del uso del portafolio electrónico en los alumnos del nivel superior. Revista Iberoamericana de Educación, 55, 173-187.

Ferla, J., Valcke, M., \& Schuyten, G. (2008). Relationships between student cognitions and their effects on study strategies. Learning and Individual Differences, 18 (2), 271-278.

Freire, L., \& Duarte, A. (2010). Concepções de aprendizagem em estudantes universitários brasileiros. Psicologia USP, 21 (4), 875898.

Freire, L. G., \& Duarte, A. M. (2012). Concepções de e autoregulação da aprendizagem do desenho artístico em estudantes universitários brasileiros. Comunicação apresentada em II Seminário de Investigação em Psicologia da Faculdade 
de Psicologia da Universidade de Lisboa, Lisboa, Portugal. Recuperado: 15 mar. 2012. Disponível: https://sites.google.com / site/programainvestigacao/3-estudos.

García-Ros, R., \& Pérez-González, F. (2011). Validez predictiva e incremental de las habilidades de autorregulación sobre el éxito académico en la universidad. Revista de Psicodidactica, 16 (2), 231-250.

Hernández Pina, F., \& Sánchez, J. (2011). Las creencias y las concepciones. Perspectivas complementarias. REIFOP, 14 (1), 165-175.

Hernández Pina, F., Fonseca, S., Rosário, P., \& Tejada, J. (2010). Impacto de un programa de autorregulación del aprendizaje en estudiantes de grado. Revista de Educación, 353, 571-588.

Horta, P. (2010). A microanálise através da entrevista semi-directiva. uma avaliação do ciclo da auto-regulação da aprendizagem. Dissertação de Mestrado, Faculdade de Psicologia da Universidade de Lisboa, Lisboa.

Martínez Fernández, J. (2007). Concepción de aprendizaje y estrategias metacognitivas en estudiantes universitarios de psicología. Anales de Psicología, 23 (1), 7-16.

Marton, F. (1981). Phenomenography - a research approach to investigating different understandings of reality. Journal of Thought, 21 (3), 28-49.

Marton, F., Dall'Alba, G., \& Beaty, E. (1993). Conceptions of learning. International Journal of Educational Reserch, 19 (3), 277-300.

Marton, F., \& Booth, S. (1997). Learning and awareness. Mahwah: Lawrence Erlbaum.

Marton, F., \& Säljö, R. (1976). On qualitative differences in learning. I. Outcome as a function of the learner's conception of the task. British Journal of Educational Psychology, 46, 115-127.

Matos, R., Pineda, Y., \& Vásquez, A. (2010). El aprendizaje del arte. Un modelo de mediación basado en la interacción sociocultural. Revista de Investigación, 69 (34), 179-207.

Meira, M., \& Pillotto, S. (2011). Arte, afeto e educação: a sensibilidade na ação pedagógica. Porto Alegre: Editora Mediação.

Miñano, P., \& Castejón, J. (2011). Variables cognitivas y motivacionales en el rendimiento académico en lengua y matemáticas: un modelo estructural. Revista de Psicodidactica, 16 (2), 203-230.

Padilla, R. (2007) El dibujo del natural. La época de la postacademia. Madrid: Ediciones Akal.

Palacio, M., Álvarez, J., \& Dorantes, M. (2010). Evaluación del aprendizaje autorregulado en estudiantes de bachillerato mexicanos. Aula Abierta, 38(1), 59-70.
Pintrich, P. (2000). Multiple goals, multiple pathways: The role of goal orientation in learning and achievement. Journal of Educational Psychology, 92 (3), 544-555.

Pozo, J., Scheuer, N., Mateos, M., \& Echeverría, M.P. (2011). Las teorías implícitas sobre el aprendizaje y la enseñanza. Em J. Pozo, N. Scheuer, M. Echeverría, M. Mateos, L. Martín, \& M. de la Cruz (Orgs.), Nuevas formas de pensar la enseñanza y el aprendizaje (pp. 95-132). Barcelona: Editorial Graó.

Purdie, N., Hattie, J., \& Douglas, G. (1996). Student conceptions of learning and their use of self-regulated learning strategies: a crosscultural comparison. Journal of Educational Psychology, 88 (1), 87-100.

Rebelo, I., \& Duarte, A. (2012). Concepções de aprendizagem com o computador em estudantes universitários. Revista da Associação Portuguesa de Psicologia, 26(2), 87-111.

Rendeiro, A., \& Duarte, A. (2007). Concepções de aprendizagem face à avaliação em estudantes do ensino secundário. Em A. Simão, A. Silva, \& I. Sá (Orgs.), Auto-regulação da aprendizagem: das concepções às práticas_(pp. 63-92). Lisboa: ED - Ui \& DCE.

Rosário, P., \& Almeida, L. (1999). As concepções e as estratégias de aprendizagem dos alunos do secundário. Actas da VI Conferência Internacional sobre Avaliação Psicológica: Formas e Contextos (pp. 713-722). Braga: APPORT/Psiquilíbrios.

Rosário, P., Nunes, T., Magalhães, C., Rodrigues, A., Pinto, R., \& Ferreira, P. (2010). Processos de auto-regulação da aprendizagem em alunos com insucesso no $1 .^{\circ}$ ano de universidade. Revista Semestral da Associação Brasileira de Psicologia Escolar e Educacional, 14(2), 349-358.

Scheuer, N., Pozo, de la Cruz, M., \& Echenique, M. (2011). Las concepciones de los niños acerca del aprendizaje del dibujo como teorías implícitas. Em J. Pozo, N. Scheuer, M. Echeverría, M. Mateos, L. Martín, \& M. de la Cruz (Orgs.), Nuevas formas de pensar la enseñanza y el aprendizaje (pp. 135-152). Barcelona: Editorial Graó.

Soto, P., \& Macías, F. (2011). Experiencia educativa en arte visual diseñada bajo un modelo de autorregulación del aprendizaje con estudiantes universitarios. RMIE, 16(49), 597-624.

Torrado, J., \& Pozo, J. (2011). Del dicho al hecho: de las concepciones sobre el aprendizaje a la práctica de la enseñanza de la música. Em J. Pozo, N. Scheuer, M. Echeverría, M. Mateos, L. Martín, \& M. de la Cruz (Orgs.), Nuevas formas de pensar la enseñanza y el aprendizaje (pp. 205-228). Barcelona: Editorial Graó.

Van den Brink, K. (2006). Conceptual relations between self-regulated learning and approaches to learning - a cross-cultural research with portuguese and german computer science students. Tese de doutorado, Universidade de Klobenz-Landau, Klobenz. 
Winner, E., \& Hetland, L. (2006). The arts and academic achievement: What the evidence shows. Journal of Aesthetic Education, 34, 3-11.

Yang, Y.-F., \& Tsai, C.-C. (2010). Conceptions of and approaches to learning through online peer assessment. Learning and Instruction, 20, 72-83.

Zimmerman, B. (1998). Developing self-fulfilling cycles of academic regulation: an analysis of exemplary instructional models. Em D. Schunk, \& B. Zimmerman (Orgs.), Self-regulated learning: from teaching to self-reflective practice (pp. 1-19). New York: The Guilford Press.
Zimmerman, B. J. (2000). Attaining self-regulation: a social cognitive perspective. Em M. Boekaerts, P. Pintrich \& M. Zeidner (Orgs.). Handbook of self-regulation (pp. 13-40). San Diego: Academic Press.

Zimmerman, B. (2008). Investigating self-regulation and motivation: historical background, methodological developments, and future prospects. American Educational Research Journal, 45(1), 166 $-183$.

Zimmerman, B., \& Martinez-Ponz, M. (1986). Development of a structured interview for assessing student use of self-regulated learning strategies. American Educational Research Journal, 23(4), 614-628.

Recebido em: 23/01/2013

1a. Reformulação em: 22/08/2013

2a. Reformulação em: 05/05/2014

Aprovado em: 21/05/2014

\section{Sobre o autor}

Luiz Gustavo Lima Freire (luizgustavolfreire@ig.com.br)

Doutorando em Psicologia da Educação pela Universidade de Lisboa, Faculdade de Psicologia; bolsista de investigação científica pela Fundação de Apoio à Ciência e à Tecnologia.

O autor agradece o apoio prestado pela Fundação de Apoio à Ciência e à Tecnologia 